\title{
Post-CMOS Compatible Microfabrication of a Multi- Analyte Bioelectrochemical Sensor Array Microsystem
}

\author{
Yue Huang, Andrew Mason, Aaron J. Greiner, Robert Y. Ofoli and R. Mark Worden \\ Michigan State University, East Lansing, MI 48824, USA \\ huangyu3@egr.msu.edu
}

\begin{abstract}
A multi-analyte electrochemical sensor array platform has been developed for protein-based biosensors utilizing post-CMOS compatible fabrication procedures that enables formation of single-chip biosensor array microsystems. The process was developed on a glass substrate to emulate the surface of a CMOS chip. A three-electrode system, including an array of gold working electrodes (WE) and an on-chip $\mathrm{Ag} / \mathrm{AgCl}$ reference electrode (RE), was formed to facilitate electrochemical analysis protein-based interfaces. SU-8 epoxy resin was subsequently applied and patterned as an insulation and planerization layer before application of microfluidic channels used to self-assemble novel bio-interfaces on individual working electrodes. The protein-based biointerfaces provide selectivity, and the electrode floorplan maximizes sensitivity and minimizes solution resistance errors. The electrodes on the prototype platform can be easily scaled to form $\sim 100$ electrodes on the surface of a $10 \mathrm{~mm}^{2}$ CMOS chip.
\end{abstract}

\section{INTRODUCTION}

Simultaneous detection and quantification of multiple analytes in a continuous use, real-time sensor platform is appealing for industrial, environmental and biosecurity applications. In previous work we have demonstrated novel nanostructured bioelectronic and biomimetic interfaces that tether proteins to gold electrodes [1], and have presented a multi-channel CMOS potentiostat chip [2]. This paper describes a post-CMOS fabrication procedure suitable for combining the bio-interfaces with CMOS sensor interface circuits as an intermediate step toward fully integrated nanostructured biosensor array microsystems.

\section{POST-CMOS COMPATIBLE MiCROFABRICATION}

\section{A. Planar Three-Electrode Electrochemical System}

In analytical electrochemical systems, three electrodes are preferred over two electrodes, which are typically limited to potentiometric device applications. For analytical purpose, knowing the potential of the working electrode is important for characterizing the redox species in an electrochemical reaction. Since the voltage drop at each electrode/solution interface and across the electrolyte is dependent on potential, current, and frequency, it is not practical to trust the working electrode potential as measured from the auxiliary electrode in a two electrode system [2]. By adding a reference electrode with a constant potential, the applied potential on the working electrode can be more precisely obtained.

This work was supported by the Michigan Economic Development Corp through the Technology Tri-Corridor fund.
For reliable connection to chip-scale circuitry without the noise introduced by external wiring, the electrodes can be fabricated on surface of the chip. Figure 1 illustrates the structure of the planar electrode that has been developed using a post-CMOS compatible fabrication process. Although the size and shape of the electrode can be easily modified in the post-CMOS patterning mask, a disk-shaped working electrode is used to reduce the background current due to isotropic diffusion compared to bar electrodes. This provides better signal to noise ratio and improves the limit of detection. While the shape of the working electrode is important, the shape of counter and reference electrodes do not play a major role in performance. However, their position relative to the working electrode is a significant consideration.

To develop a CMOS compatible process resulting in electrode arrays that facilitate experimental study of novel bio-interfaces, a macro-scale prototype was designed to ease subsequent processing steps and simplify device handling. As shown in Figure 2, the prototype array has four $2 \mathrm{~mm}$ working electrodes and four reference electrode sites that are electrically connected to each other surrounded by a field of metal acting as the counter electrode with circular opening at four locations through the passivation layer. The fabrication processes used to create the array are fully scalable and can be applied to create electrodes on the order of $1 \mu \mathrm{m}$ diameter. In the next generation of devices, once the processes for forming bio-interfaces on the electrodes have been optimized, the working electrodes will be scaled down.

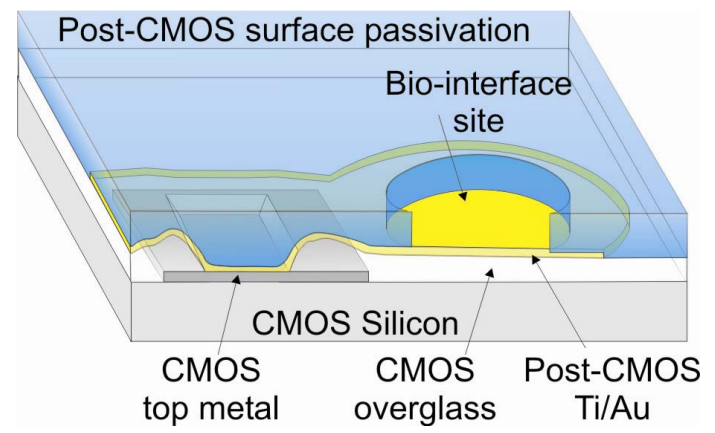

Figure 1. Details of the passivated electrode and its connection to top CMOS metal pad via overglass opening. 


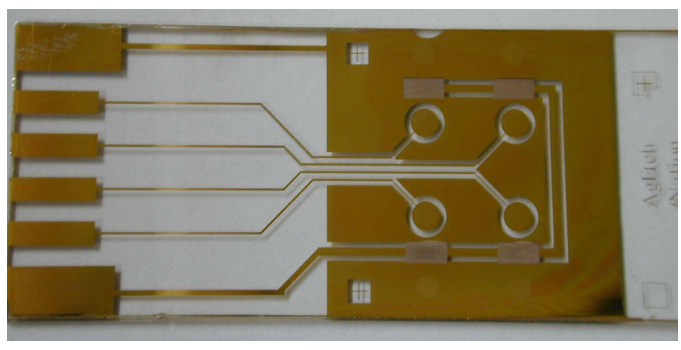

Figure 2. Microfabricated electrode array on glass slide, with $2 \times 2$ disk working electrode array, counter electrode and on-site reference electrodes.

Double layer capacitance at the electrode interface decreases as the square of electrode size, causing current density to increase and non-linear diffusion across the electrode to dominate. For example, using ultramicroelectrodes, the measurement of chemical concentrations can be made even in highly resistive solutions within microsecond time scales [3], which is difficult or impossible with conventional macro-scale electrodes. However, because connecting ultramicroelectrodes to external instrumentation limits performance, the best way to read out such devices is to integrate the potentionstat directly on chip with the electrodes, as provided in our approach.

Polycrystalline gold thin films were chosen as the working electrode material because they provide a suitable substrate for the formation of self-assembled monolayers (SAMs) from thiols, sulfides, and disulfides. These SAMs can be used as intermediate layers for the coupling of a wide range of molecules, including enzymes and antibodies. Other excellent electrode materials like diamond were not considered because of their incompatibility with post-CMOS fabrication constraints, such as low processing temperatures.

\section{B. Thin Film Deposition}

Physical vapor deposition (PVD) processes form thin film layers by evaporating or glow discharging atoms from a source and then condensing or depositing the material onto the substrate surface. Normally, evaporation gives a better surface smoothness than sputtering. Surface roughness is important for the quality of subsequent bio-interface formation. Microscopic roughness and the presence of crystal grain boundaries can have a distinct effect on the density of defects in SAMs [4]. In our experiments, we used an Edward 306 Thermal Evaporator for depositing titanium, gold and silver. A tantalum boat was used for the titanium adhesion layer, and an alumina-coated molybdenum boat was used for gold and silver. The alumina protective coating is inert with gold and silver metals, and neither gold nor silver wet the alumina, which eliminates resistance changes in the boat during melting. Due to their non-wetting characteristics, gold and silver form spheres when melted, resulting in "point source" performance. To achieve good deposition quality, avoiding cross contamination is as important as using high purity materials. This is achieved by dedicating a compartment in the PVD system to each metal that is separated by dividers on the source stage.

To be post-CMOS compatible, deposition temperature on the substrate should be lower than $450^{\circ} \mathrm{C}$ to avoid melting the CMOS aluminum alloy metals or changing the electrical properties of CMOS transistors. To verify our process achieved this goal, we first tried to vent the PVD system immediately after deposition and test the sample temperature with an infrared thermometer. The average temperature reading was $90^{\circ} \mathrm{C}$. However, the actual peak temperature is expected to be higher because of heat dissipation losses through the conductive aluminum substrate holder during venting. Unable to determine a method for testing the actual peak temperature of the substrate in the vacuum chamber, the temperature was examined indirectly. By putting materials with known deformation temperatures in place of the sample and observing if deformation occurred after deposition, we concluded that the peak process temperature was between $130^{\circ} \mathrm{C}$ (where a generic polyester tape started to deform) and $160^{\circ} \mathrm{C}$ (where Scotch ${ }^{\circledR}$ Magic ${ }^{\mathrm{TM}}$ Tape 810 would start to deform).

The base pressure at the start of deposition was $2 \times 10^{-6}$ Torr. A high vacuum environment allows for higher film material purity and lowers the required processing temperatures. For example, the melting point of titanium is $1660^{\circ} \mathrm{C}$ at atmospheric pressure and only $1235^{\circ} \mathrm{C}$ at $2 \times 10^{-6}$ Torr.

Titanium and gold were deposited on a clean substrate of $\mathrm{SiO}_{2}$-covered silicon with openings for electric connections as shown in Figure 1. The titanium layer was $30 \AA$ thick and acts as an adhesion layer between gold and the oxide layer. For deposition of titanium, current was brought up to 3.8 amps gradually, until the deposition rate reached $0.1 \AA / \mathrm{sec}$. $100 \mathrm{~nm}$ of gold was deposited at $2.4 \mathrm{amps}, 0.8 \AA / \mathrm{sec}$.

\section{Photolithography}

Photolithography is a fabrication technique for the transfer of patterns through light energy from photomasks to substrates coated with light-sensitive photoresist. Shipley Microposit S1813 positive photoresist was spin-coated over the $\mathrm{Ti} / \mathrm{Au}$ deposited on glass slides at approximately 4000rpm to achieve a $1.3 \mu \mathrm{m}$ thickness. The slide was soft baked for 10 minutes at $95^{\circ} \mathrm{C}$ and then exposed to an $18.4 \mathrm{~mW} / \mathrm{cm}^{2}$ UV source for 9.5 second with AB-M mask aligner. After a hard bake of 20 minutes, the sample was developed in Microposit 452 developer for 40 to 60 seconds with mild agitation. It was then rinsed in DI water for 1 minute and dried with nitrogen.

To release the Ti/Au pattern, the sample was placed in a 1:4 (by volume) solution of $\mathrm{KI}$ and $\mathrm{I}_{2}$, respectively. After the pattern was released, it was rinsed with DI water and then placed in a 1:20 (by volume) solution of $\mathrm{HF}$ and $\mathrm{H}_{2} \mathrm{O}$, 
respectively, for 10 seconds. It was then rinsed again with DI water. Overexposure to these etching solutions was avoided to reduce defects or problems with the pattern peeling off. After patterning the Ti/Au layer, a lift-off process was used to pattern a 500nm silver layer for the reference electrodes.

\section{Fabrication of $\mathrm{Ag} / \mathrm{AgCl}$ Reference Electrode}

$\mathrm{An} \mathrm{Ag} / \mathrm{AgCl}$ reference electrode was chosen over other reference electrodes because it can readily be miniaturized, it is not easily contaminated by the steps required to form biointerfaces, and it is safe for in vivo applications. Although a bare $\mathrm{Ag} / \mathrm{AgCl}$ electrode is not stable in an aqueous solution for extended periods of time without a special coatings like Nafion, it can still function in a small amount of solution using microfluidic channels for a short period of time [5]; depend on the analyte in the solution and the abundance of silver and silver chloride in the reference electrode.

To form silver chloride, silver can be reacted by electrochemical anodization in $\mathrm{HCl}$ or chemical oxidation with aqueous $\mathrm{FeCl}_{3}$. Reference electrodes made by both methods have equal stabilities, but the latter behaves more Nernstian [5]. After silver was patterned on the array, a 1 $\mathrm{mM}$ solution of $\mathrm{FeCl}_{3}$ was applied for 10 seconds, followed by a rinse with DI water. Chlorination with ferric chloride is easy to perform and produces a uniform layer of $\mathrm{AgCl}$. The electrochemical potential established by this $\mathrm{Ag} / \mathrm{AgCl}$ electrode is given by the following equilibrium reaction:

$$
\mathrm{AgCl}(\mathrm{s})+\mathrm{e} \leftrightarrow \mathrm{Ag}(\mathrm{s})+\mathrm{Cl}-
$$

The potential is then set by Nernst equation [6]:

$$
\mathrm{E}_{\mathrm{Ag}, \mathrm{AgCl}}^{0}=\mathrm{E}_{\mathrm{Ag}, \mathrm{Ag}^{+}}^{0}+\mathrm{RT} \ln \mathrm{K}_{\mathrm{s}} / \mathrm{F}
$$

where $E_{A g, A g C l}$ is the actual potential of cell, $E_{A g, A g+}^{0}$ is the standard potential, $R$ is the ideal gas constant, $T$ is absolute temperature, $n$ is number of electrons transferred in the electrochemical reaction, $F$ is Faraday's constant, and $K_{s}$ is the solubility constant. The electrochemical potential, therefore, depends on the concentration of chloride ions.

The reference electrode was tested after fabrication in a $3 \mathrm{M} \mathrm{KCl}$ solution. Compared with commercial $\mathrm{Ag} / \mathrm{AgCl}$ electrodes, it was found to have a $50 \mathrm{mV}$ potential difference. Figure 3 illustrates the final electrode array device including subsequently applied layers with microfluidic channels.

\section{Bioelectrochemical SENSOR ARray}

The $2 \times 2$ electrode array prototype was constructed with three electrodes (WE, CE, RE) for each of the four sensor sites. The polydimethylsiloxane (PDMS) stamp, shown in Figure 4, was then made for delivering fluids necessary to form bio-interfaces on each working electrode and introducing the analyte being tested. To verify operation of the array with bioelectrochemical sensor interfaces, one of

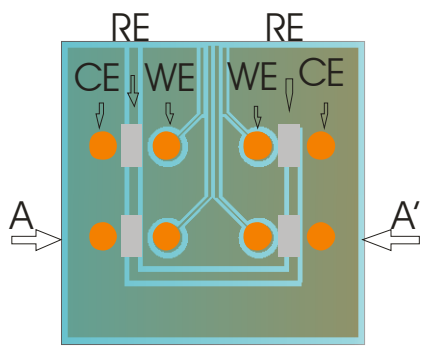

(A)

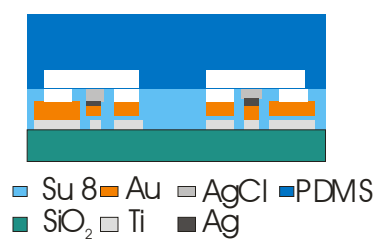

(B)
Figure 3. Electrode array. (A) Top view, (B) Cross-section view of A-A' section, with imposed PDMS stamp.

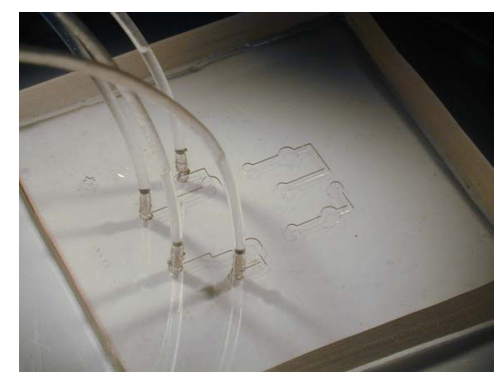

Figure. 4. PDMS microfluidic channel to form bio-interface on individual working electrodes and deliver analytes, with dedicate inlets and outlets for each channel.

the working electrodes in the array was modified by secondary alcohol dehydrogenase (sADH).

\section{A. Electrode Array Fabrication Process}

A 3-electrode electrochemical system was built on a glass slide (Figure 2), with a 2x2 $\mathrm{Ti} / \mathrm{Au}$ working electrode array, on-site $\mathrm{Ag} / \mathrm{AgCl}$ reference electrodes, and a common counter electrode. The electrode array was coated with SU-8 epoxy resin and was patterned to confine the area of WEs, to insulate metal leads, and to form a level surface for subsequent application of PDMS microfluidic channels.

\section{B. Microfluidic Channel for Modifying Electrode Array}

PDMS microfluidic channels were made to deliver reagents for modifying the working electrodes. Sylgard $^{\mathrm{TM}} 184$ PDMS from Dow Corning was used to fabricate a masterpiece in a petri dish. The master was created with a MicroChem SU-8 2050 epoxy-based negative resist on a smooth silicon wafer. The epoxy was exposed through a photomask under UV light. The resulting master structure was used as a mold to create PDMS blocks equipped with microfluidic channels. Finally, holes where tubes could be inserted were created at the inlets and outlets of the channels.

\section{Modification of Electrode}

The Au electrodes were first modified with cysteine, and then incubated in a solution of toluidine blue $\mathrm{O}$ (TBO) in $0.1 \mathrm{M}$ phosphate buffer (PBS), $\mathrm{pH}=7.4$, in the presence of $\mathrm{N}$ - 
hydroxysuccinimide (NHS). The electrodes were then reacted with 3- carboxyphenylboronic acid (CBA) and $1 \mathrm{mM}$ $\mathrm{NAD}(\mathrm{P})+$ in PBS under ambient conditions for 2 hours and washed with DI water afterward. In this step, the NAD(P)+ binds to the interface through an affinity linkage between the boronic acid ligand and the cis-diols of the NAD $(\mathrm{P})+[1]$. The TBO-NADP+ functionalized electrodes were reacted with $1 \mathrm{mg} \mathrm{mL}^{-1}$ secondary alcohol dehydrogenase (sADH) from thermoanaerobacter ethanolicus, in PBS. The resulting protein was then crosslinked by immersion in glutaric acid for $1 \mathrm{hr}$ and then washed with water. The final interface provides mediated electron transfer between the electrode and the sADH [1].

\section{TEST RESUlTS}

The electrochemical system was verified by cyclic voltammetry in $20 \mathrm{mM}$ potassium ferricyanide and $1 \mathrm{M} \mathrm{KCl}$. The resulting CV curve is plotted in Figure. 5. An electrode functionalized with sADH was subjected to cyclic voltametry with $2 \%$ isopropyl alcohol (IPA) in PBS buffer solution. Catalyzed reduction and oxidization at $-10 \mathrm{mV}$ and $220 \mathrm{mV}$, respectively, with respect to an $\mathrm{Ag} / \mathrm{AgCl}$ reference electrode, shown in Figure 6, demonstrates detection of the redox pair of IPA and acetone. The modified electrode displayed good selectivity in a multi-analyte environment with a cathode peak current of $0.1 \mu \mathrm{A}$, compared to a $130 \mu \mathrm{A}$ peak current derived from potassium ferricyanide on a bare gold. This rejection of other analytes establishes species recognition in a multi-analyte environment.

The same techniques can be used to create a wide range of biosensor elements based on tethered proteins. With the post-CMOS electrode array process established, we are now working to miniaturize the bio-interfaces to chip scale where they can be integrated with our existing CMOS potentiostat. We have also developed bio-interfaces based on membrane proteins that can be included in the array.

\section{CONCLUSION}

A post-CMOS compatible fabrication process has been developed and presented for a multi-analyte electrochemical sensor array platform utilizing nanostructured, protein-based biosensors. The prototype $2 \times 2$ gold working electrode array of a 3-electrode electrochemical system, including an onchip $\mathrm{Ag} / \mathrm{AgCl}$ reference electrode, was developed on a glass slide using techniques that can readily be implemented on the surface of a CMOS chip to create an integrated biosensor array. The array was passivated by a layer of SU8 on top of which a PDMS layer with microfluidic channels was incorporated to deliver solutions for bio-interface formation and testing. Experimental measurements of the electrode system and a subsequently formed bio-interface demonstrate successful operation of the device. The

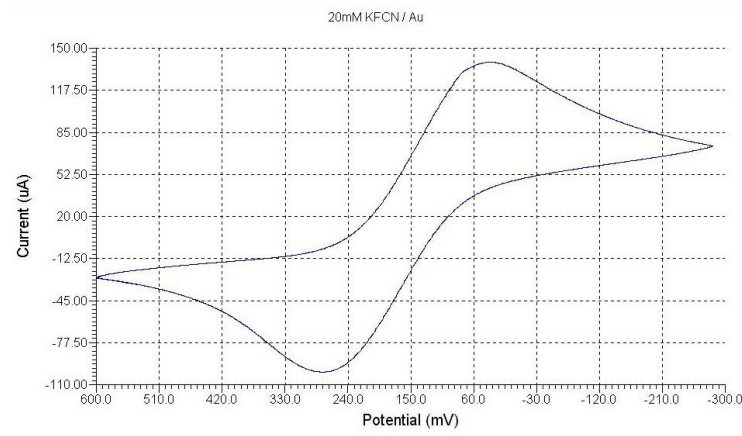

Figure 5. Cyclic voltammogram of post-CMOS electrodes in $20 \mathrm{mM}$ potassium ferricyanide, $1 \mathrm{M} \mathrm{KCl}$ at $25^{\circ} \mathrm{C}$ and $100 \mathrm{mVS}^{-1}$ scan rate, vs. $\mathrm{Ag} / \mathrm{AgCl}$ reference electrode.

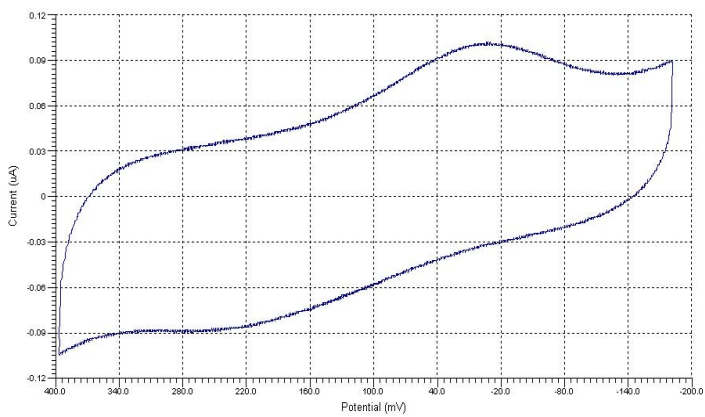

Figure 6. Cyclic voltammogram of an $\mathrm{SADH}$ enzyme modified electrode in $2 \%$ IPA PBS buffer solution at $25 \mathrm{C}^{\circ}$ and $200 \mathrm{mVS}^{-1}$ scan rate vs. $\mathrm{Ag} / \mathrm{AgCl}$ reference electrode, showing reduction and oxidation peaks.

prototype array can be readily scaled to form $\sim 100$ electrodes on the surface of a $10 \mathrm{~mm}^{2}$ CMOS chip.

\section{ACKNOWLEDGMENT}

Brian Hassler's assistance in preparing sADH modified electrodes is gratefully acknowledged.

\section{REFERENCES}

[1] B. Hassler, R. M. Worden, A. Mason, P. Kim, N. Kohli, J. G. Zeikus, M. Laivenieks, and R. Ofoli, "Biomimetic Interfaces for a Multifunctional Biosensor Array Microsystem," IEEE Int. Conf. on Sensors, Vienna, Austria, pp. 991-994, October 2004.

[2] J. Zhang, Y. Huang, N. Trombly, C. Yang, A. Mason, "Electrochemical Array Microsystem with Integrated Potentiostat," IEEE Int. Conf. on Sensors, Irving CA, pp. 385-388, October 2005.

[3] R. M. Wightman, "Voltammetry with Microscopic Electrodes in New Domains," Science, vol. 240, pp. 415-420, 1988.

[4] S. E. Creager, L. A. Hockett, and G. K. Rowe, "Consequences of Microscopic Surface Roughness for Molecular Self -Assembly," Langmuir, pp. 854-861, 1992.

[5] B. J. Polk, A. Stelzenmuller, G. Mijares, W. MacCrehan, and M. Gaitan, " $\mathrm{Ag} / \mathrm{AgCl}$ microelectrodes with improved stability for microfluidics," Sensors and Actuators B: Chemical, vol. 114, pp. 239-247, 2006.

[6] D. J. G. Ives and G. J. Janz, Reference electrodes, theory and practice. New York: Academic Press, 1961. 